\title{
Photochemical upconversion of light for renewable energy and more
}

\section{Timothy Schmidt, Rowan Macqueen}

Timothy W. Schmidt, Rowan W. Macqueen, "Photochemical upconversion of light for renewable energy and more," Proc. SPIE 9562, Next Generation Technologies for Solar Energy Conversion VI, 956202 (4 September 2015); doi: $10.1117 / 12.2195424$

SPIE Event: SPIE Optics + Photonics for Sustainable Energy, 2015, San Diego, California, United States 


\title{
Photochemical upconversion of light for renewable energy and more
}

\author{
Timothy W. Schmidt ${ }^{a}$ and Rowan W. MacQueen ${ }^{b}$ \\ ${ }^{a}$ School of Chemistry, The University of New South Wales, Sydney, NSW 2052, Australia; \\ ${ }^{b}$ School of Chemistry, The University of Sydney, NSW 2006, Australia.
}

\begin{abstract}
Photochemical upconversion has been put forward as a candidate technology to improve the light-harvesting capabilities of thin-film photovoltaic cells, by harvesting transmitted sub-bandgap light and re-radiating the absorbed energy at a usable wavelength. Efficiencies of $10 \%$ have been observed under solar-level irradiation, and up to $86 \%$ (quantum yield of $43 \%$ ) has been observed under strong irradiance. In this proceeding, we explain the triplet-triplet annihilation mechanism underlying photochemical upconversion and delve into the chemical kinetics to extract strategies to improve device performance. We suggest that one of these strategies, concentrating the sensitizer species, may be flawed without proper consideration of the sensitizer identity, due to enhanced emitter triplet decay caused by the external heavy atom effect.
\end{abstract}

Keywords: Triplet-triplet annihilation, up-conversion, photochemistry, triplet states, delayed fluorescence

\section{INTRODUCTION}

Photochemical upconversion (PUC) achieved by sensitized triplet-triplet annihilation (TTA) in organic molecules presents many opportunities in applied photon science. ${ }^{1-5}$ In PUC, the material or chemical composition is irradiated at a longer wavelength than the ensuing (delayed) fluorescence. As such, it has potential applications in areas including: Photovoltaics, in recovering below-bandgap photon losses $;{ }^{6-10}$ Photoelectrochemistry, similarly generating more usable photons; ${ }^{11-13}$ Biological Imaging, in circumventing autofluorescence ${ }^{14-17}$ Drug Activation, stimulating drug release inside the body through the tissue window; ${ }^{18,19}$ and Water Purification, generating short wavelengths to degrade water impurites. ${ }^{20}$

The TTA mechanism is depicted in Figure 1. It proceeds with the absorption of low energy photons by a sensitizer species which is designed or chosen to have a small $S_{1}-T_{1}$ gap not less than several $k_{B} T$, and a high triplet yield. ${ }^{21}$ The high triplet yield is usually driven by inclusion of a heavy atom, such as palladium, in a sensitizer such as a metalloporphyrin. The sensitizer must have a triplet state with sufficient longevity such that it can be quenched by triplet-accepting emitter molecules at moderate concentrations $(\sim \mathrm{mM})$. The emitter species, often a modified polyacene or rylene species, is placed in its triplet state, and ideally interacts with another triplet emitter before the otherwise inevitable non-radiative triplet decay.

The interaction of two triplet molecules yields nine spin-states. These may be classified into five components of a quintet, three components of a triplet, and a single singlet component. ${ }^{22,23}$ The singlet component is capable of placing an emitter in its $S_{1}$ state, while the other is demoted to its ground state, $S_{0}$, providing that the energy of two $T_{1}$ triplets exceeds that of the lowest singlet. The $S_{1}$ emitter promptly fluoresces, ideally in high yield, to generate the upconverted fluorescence. The other spin-manifolds represent possible loss-channels. It is likely, but not inevitable, that the $T_{2}$ state is also exceeded by the energy of two $T_{1}$ molecules. In this case, the upconverted $T_{2}$ state molecule rapidly decays to $T_{1}$, while the other is quenched to $S_{0}$. It is rare that quintet molecular states are accessible at the moderate (few eV) energies involved in PUC and as such these are usually ignored. With an open triplet channel, $40 \%$ of triplet emitters can take part in upconversion, but there is no limit if only the singlet channel is in operation, giving rise to a maximum quantum yield of $50 \%$, corresponding to $100 \%$ TTA efficiency. The efficiency of the TTA process is described by the parameter $\eta_{c}$, the proportion of triplets which

Further author information: (Send correspondence to T.W.S.)

T.W.S.: E-mail: timothy.schmidt@unsw.edu.au, Telephone: 61293854653

Next Generation Technologies for Solar Energy Conversion VI, edited by Oleg V. Sulima,

Gavin Conibeer, Proc. of SPIE Vol. 9562, 956202 - (c) 2015 SPIE

CCC code: $0277-786 \mathrm{X} / 15 / \$ 18 \cdot$ doi: $10.1117 / 12.2195424$

Proc. of SPIE Vol. $9562956202-1$ 


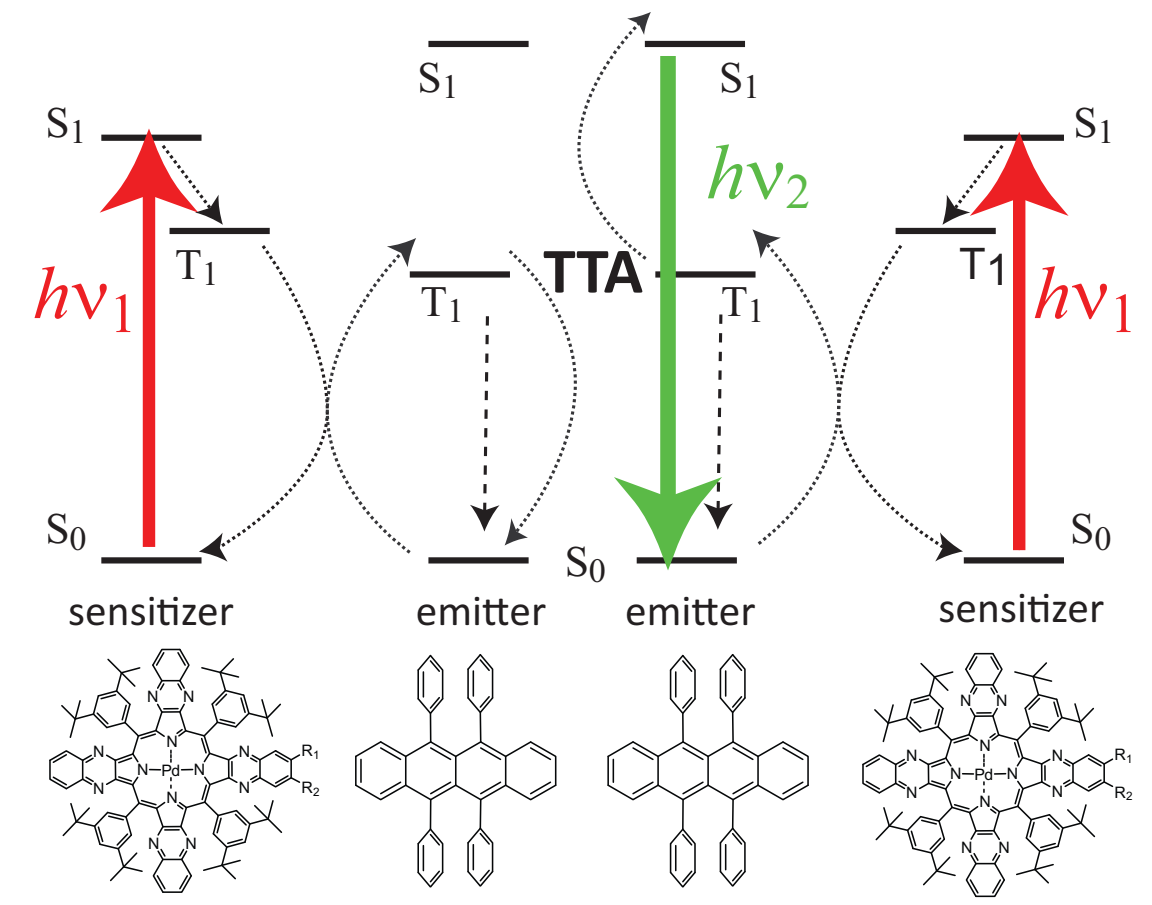

Figure 1. The mechanism of PUC. Sensitizer molecules, such as the porphyrins shown, absorb low energy photons and then undergo intersystem crossing to their lowest triplet state. The triplet state energy is transferred to emitter molecules, which then undergo TTA to generate the excited singlet species which emits upconverted fluorescence.

participate in giving rise to excited singlets and thus possible photons. Rubrene has been determined to have a partially open triplet channel, with $\eta_{c} \sim 0.6$, but perylene has been shown to have $\eta_{c} \sim 1 .{ }^{22,24}$

There remain few examples of efficient emitters compatible with state-of-the-art thin film solar cells. Rubrene has thus been implemented by our group in experiments with a range of devices, including a-Si:H cells, OPV, and DSCs. ${ }^{6-10}$ A rigorous lock-in experiment demonstrated that, under one-sun illumination, rubrene was capable of only $1 \%$ TTA efficiency when sensitized by our bespoke $\mathrm{PQ}_{4} \mathrm{Pd}$ metalloporphyrin in toluene. ${ }^{25}$ This poor performance can be attributed to tardy diffusion giving rise to a poor triplet-triplet annihilation rate, spurring the search for faster, efficient emitters in the yellow-green region of the spectrum. It also motivates improvements in TTA-device efficiency by rigorous inspection of the chemical kinetics involved in this photochemical process.

In the following, we review the basic kinetics of the TTA-PUC process, ${ }^{21}$ arriving at some simple expressions which suggest four main strategies with which to improve upconversion efficiencies. We address these in turn, with reference to the literature, but dwell on the last of these, the concentration of sensitizers, and reveal a new design principle taking into account the external heavy atom effect engendered by sensitizers containing heavy metals.

\section{KINETIC MODEL}

The rate law governing the decay of emitter triplet states is

$$
\frac{d\left[{ }^{3} E\right]}{d t}=0=k_{\phi}[S]-k_{1}\left[{ }^{3} E\right]-k_{2}\left[{ }^{3} E\right]^{2},
$$

where $[X]$ is the concentration of species $X$, and $S$ and $E$ denote the sensitizer and emitter species respectively. The rate of photon $(\phi)$ emission per unit volume is proportional to the second-order term, corrected for a conversion factor which accounts for unsuccessful triplet encounters,

$$
\frac{d[\phi]}{d t}=\frac{k_{2}\left[{ }^{3} E\right]^{2}}{2} \eta_{c}
$$


where the factor of two accounts for two triplets creating a single photon in a successful encounter. The goal of photochemical upconversion is to bring about efficient conversion, in which case $k_{2}\left[{ }^{3} E\right]>k_{1}$. Since this is not yet achieved, it being the goal of the present studies, we make the assumption that $k_{1} \gg k_{2}\left[{ }^{3} E\right]$, which is equivalent to being far below the threshold pumping rate as described by Monguzzi and co-workers. ${ }^{26}$ Under the assumption of inefficient operation, we find that

$$
\left[{ }^{3} E\right]=\frac{k_{\phi}[S]}{k_{1}} .
$$

The rate of upconversion is then

$$
\frac{d[\phi]}{d t}=\frac{k_{2}\left(k_{\phi}[S]\right)^{2}}{2 k_{1}^{2}} \eta_{c}
$$

This immediately leads to three factors that might lead to higher triplet concentrations and thus more efficient upconversion, where the relative efficiency is given by

$$
f=\frac{k_{2}\left[{ }^{3} E\right]}{k_{1}+k_{2}\left[{ }^{3} E\right]},
$$

being the proportion of decay events due to second order processes.

Those factors are: increase $k_{2}$; decrease $k_{1}$; increase $k_{\phi}$; and increase $[S]$. These factors are discussed in turn below.

\section{FASTER ANNIHILATION}

The rate of triplet-triplet annihilation has an upper limit related to the diffusion controlled reaction limit which is about $10^{10} \mathrm{M}^{-1} \mathrm{~s}^{-1}$ in toluene. The diffusion limit is calculated from

$$
k_{d}=\frac{8 R T}{\eta},
$$

where care must be taken with regards to units. ${ }^{21}$ The upper limit on $k_{2}$, with no regard to spin-statistics, is $2 k_{d}$, since two triplets are removed per successful encounter. However, most annihilators (emitters) exhibit values for $k_{2}$ which are considerably smaller than $k_{d}$. For example, 9,10-diphenylanthracene exhibits $k_{2} \sim 2 \times 10^{9} \mathrm{M}^{-1} \mathrm{~s}^{-1}$ in toluene, while rubrene achieves only $k_{2} \sim 1 \times 10^{8} \mathrm{M}^{-1} \mathrm{~s}^{-1}$ in the same solvent. ${ }^{22}$ Rubrene is bulky, which contributes to slow diffusion. The intimate $\pi-\pi$ contact required for TTA to proceed is also sterically hindered, and these two factors conspire to cause tardy TTA. ${ }^{27}$ It should be noted, though, that fast is not always better. If the triplet channel is open, then three-quarters of the TTA events are dark, and the upconversion yield is slashed by $60 \%$. This is the case for 9-chlorobisphenylethynylanthracene, which despite exhibiting rapid TTA, with $k_{2} \sim 6 \times 10^{9} \mathrm{M}^{-1} \mathrm{~s}^{-1}$ in $\operatorname{DMF}\left(k_{d} \sim 8 \times 10^{9} \mathrm{M}^{-1} \mathrm{~s}^{-1}\right)$, was found to exhibit a rather low $\eta_{c}{ }^{28}$

\section{SLOWER EXCITED-STATE DECAY}

It has long been known that non-radiative rates in aromatic hydrocarbons, in the weak-coupling limit, can be significantly diminished by (per)deuteration. The effect is ascribed to lower Franck-Condon factors for the energy-accepting modes. In the case of hydrocarbons, since C-H stretches are the highest frequency modes and require a smaller change in number of quanta upon intersystem crossing than other modes such as C-C stretches, they are considered the primary energy-accepting modes. Deuteration decreases the frequencies by a factor of $\sqrt{2}$, and thus increases the effective energy gap by the same factor. The non-radiative rates thus diminish in the deuterated sample. We recently reported a $45 \%$ increase in upconversion efficiency in the weak pumping regime in a composition with perdeuteroperylene as the emitter as compared to the hydrogenated species. ${ }^{29}$ However, much more dramatic effects on triplet lifetimes, up to factors of 10, have been observed in other compounds from $77 \mathrm{~K}$ to room temperature, suggesting that indeed this strategy will serve to improve upconversion efficiencies. ${ }^{30}$ 


\section{LIGHT CONCENTRATION}

The goal of photochemical upconversion for renewable energy is to enhance the electrical output of thin-film solar cells under solar irradiation. As such, external concentration of light is not deemed relevant to the present studies. However, light may be concentrated internally by use of micro-optics, such as parabolic reflectors or microlens arrays. The latter requires an optical disconnect between the lenses and the upconvertor, and lenses with device-scale focal lengths. Such a device is not an impossibility, but its practicality is yet to be investigated. We investigated a field of parabolic reflectors as a backing to an upconvertor, optimizing the upconvertor thickness by means of a half-cuvette with a micrometer-driven adjustable substrate. While our experimental realization yielded an upconversion output 2.25 that of a deep cuvette, ray-tracing models indicated that a nine-fold increase should be possible for an optimally engineered device. ${ }^{8}$

\section{CONCENTRATED SENSITIZERS}

Increasing $[S]$ would appear an obvious strategy to achieve more efficient upconversion, and in principle it would appear that such a strategy would be facile to implement. However, there are solubility limits to metalloporphyrins of the sort implemented as sensitizers in our study. Indeed, some researchers have found that aggregation of sensitizers caused up to $97 \%$ loss of triplet states. ${ }^{31}$ However, as will be shown below, such a strategy should be approached with caution.

The sensitizers chosen in upconversion studies are almost always metalloporphyrins. The have a small but large enough singlet-triplet energy gap and very efficient intersystem crossing, largely due to the internal heavy atom effect. But, in concentrated solutions of sensitizer, approaching $0.1 \mathrm{M}$, the collision rate of a triplet emitter with ground state sensitizers will begin to compete with the natural decay. Diffusion-limited collisions will proceed at approximately $10^{10} \mathrm{M}^{-1} \mathrm{~s}^{-1}$ in solvents such as toluene, resulting in collision rates of $10^{9} \mathrm{~s}^{-1}-$ one per nanosecond! Most studies are performed with concentrations of sensitizer nearer the $10^{-4} \mathrm{M}$ mark, bringing collisions into the microsecond regime. If one in a thousand collisions results in quenching the emitter triplet through the external heavy atom effect, $k_{q} \sim 10^{7} \mathrm{M}^{-1} \mathrm{~s}^{-1}$, then the effect would be hardly noticeable under most conditions. ${ }^{32}$

Taking external collisions with heavy atoms into account, we re-write the above equations to give

$$
\frac{d\left[{ }^{3} E\right]}{d t}=0=k_{\phi}[S]-\left(k_{1}+k_{q}[S]\right)\left[{ }^{3} E\right]-k_{2}\left[{ }^{3} E\right]^{2} .
$$

and

$$
\frac{d[\phi]}{d t}=\frac{k_{2}\left(k_{\phi}[S]\right)^{2}}{2\left(k_{1}+k_{q}[S]\right)^{2}} \eta_{c} .
$$

This suggests an upper limit for the rate of upconversion per unit volume of

$$
\lim _{[S] \rightarrow \infty} \frac{d[\phi]}{d t}=\frac{k_{2} k_{\phi}^{2}}{2 k_{q}^{2}} \eta_{c} .
$$

However, the total amount of upconversion is an integral over the irradiated volume. For a deep cuvette, without a back-reflector, ${ }^{7}$ the total amount of upconversion per unit time is

$$
W_{U C}=A \int_{0}^{\infty} d z \frac{d[\phi]}{d t}
$$

where

$$
k_{\phi}(z)=k_{\phi}(0) \exp (-\epsilon[S] z),
$$

and $A$ is the illuminated area. The $z$-dependence of $k_{\phi}$ gives

$$
W_{U C}=A \frac{k_{2}\left(k_{\phi}(0)[S]\right)^{2}}{4 \epsilon[S]\left(k_{1}+k_{q}[S]\right)^{2}} \eta_{c},
$$




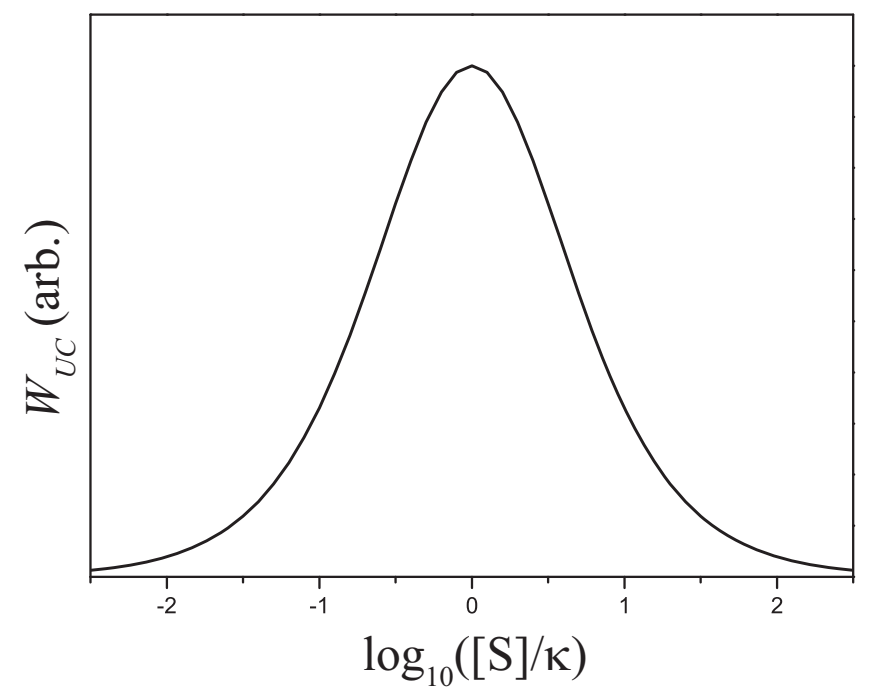

Figure 2. The upconversion output of a deep cuvette as a function of the ratio of the sensitizer concentration, $[S]$, to $\kappa$, the ratio of the natural emitter triplet decay to the triplet quenching due to sensitizer molecules, $k_{1} / k_{q}$.

which suggests an upper limit for $W_{U C}$. The inverse dependence on the molar extinction coefficient (base- $e$, non-decadic), $\epsilon$ is misleading - both the parameter $k_{\phi}$ and the molar extinction coefficient are proportional to the absorption cross section, $\sigma$. As such, the total upconversion rate is proportional to $\sigma$. Combining most of the constants into a single variable, we simplify the expression to

$$
W_{U C} \propto \frac{[S]^{2}}{[S](\kappa+[S])^{2}},
$$

where $\kappa=k_{1} / k_{q}$, with dimensions of concentration. The maximum of this function occurs at

$$
\frac{\kappa-[S]}{(\kappa+[S])^{3}}=0
$$

A plot of $W_{U C}$ as a function of $[S] / \kappa$ is given in Figure 2. The maximum upconversion rate for a cuvette without a back-reflector occurs at $[S]=\kappa=k_{1} / k_{q}$. For an emitter such as rubrene, with $k_{1} \sim 10^{4} \mathrm{~s}^{-1}$, this implies that, if $k_{q}$ is as little as $10^{7} \mathrm{M}^{-1} \mathrm{~s}^{-1}, 32$ then the optimal concentration of sensitizer is $[S]=10^{-3} \mathrm{M}$, which is similar to that implemented in our recent studies.

We hypothesized that locally concentrating the sensitizer may allow for effectively increasing the triplet concentration without deleteriously affecting the emitter triplet lifetime. ${ }^{33}$ Sensitizer-decorated silica nanoparticles were prepared and suspended in a solution of emitter. The upconversion kinetics were followed after pulsed excitation to reveal the first-order decay constant of the emitter triplets, which is a combination of the natural decay and all quenching pathways. It was found that the nanoparticle suspension resulted in faster triplet decay, irrespective of the nanoparticle concentration, as shown in Figure 3. That the two nanoparticle suspensions resulted in similar kinetics suggests that the external heavy atom effect operates at a rate faster than the diffusion of emitter triplets away from the nanoparticles, in effect a geminate process. In future studies we will investigate whether zinc porphyrins, with lower triplet yields but much a smaller external heavy atom effect, might yield higher upconversion efficiencies than the palladium porphyrins heretofore studied.

\section{CONCLUSIONS}

Inspection of a simple kinetic model for the PUC process reveals four strategies to improve upconversion efficiency: faster annihilators; slower triplet decay; more concentrated light; and higher sensitizer concentration. Each of 


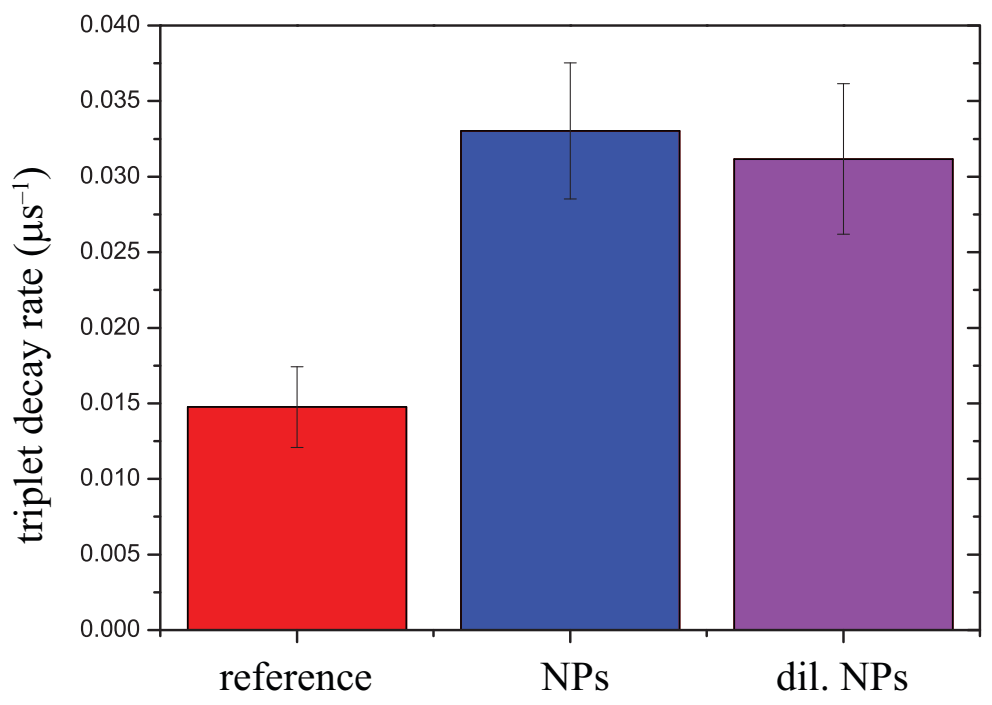

Figure 3. The observed decay rate of emitter triplets for three samples: a simple solution-based upconvertor (reference); a solution of sensitizer-decorated nanoparticles (NPs); and a dilute solution of sensitizer-decorated nanoparticles (dil. NPs).

these can be addressed in different ways. Faster annihilators demand less-bulky substituents. Slower triplet decay can be achieved through judicious phonon engineering. Light concentration can be achieved by structuring the back-reflector of the device with spherical mirrors. But, the last of these should be approached with caution. We have shown with a simple kinetic model that the external heavy atom effect can bring about severe limitations to upconversion rates, with optimal sensitizer concentrations in the $\mathrm{mM}$ range for reasonable kinetic parameters. This places limitations on the design of nanostructured upconvertors with high sensitizer concentrations.

\section{ACKNOWLEDGMENTS}

This work was funded by the Australian Renewable Energy Agency (Project A-023), with contributions from The New South Wales Government and The University of Sydney. Aspects of the research were supported under Australian Research Council's Discovery Projects funding scheme (DP110103300). RWM acknowledges the contribution from ARENA Fellowships (5-F004). TWS acknowledges the Australian Research Council for a Future Fellowship (FT130100177).

\section{REFERENCES}

[1] Singh-Rachford, T. N. and Castellano, F. N., "Photon upconversion based on sensitized triplet-triplet annihilation," Coord. Chem. Rev. 254, 2560-2573 (2010).

[2] Gray, V., Dzebo, D., Abrahamsson, M., Albinsson, B., and Moth-Poulsen, K., "Triplet-triplet annihilation photon-upconversion: Towards solar energy applications," Phys. Chem. Chem. Phys. 16(22), 10345-10352 (2014).

[3] Schulze, T. F. and Schmidt, T. W., "Photochemical upconversion: Present status and prospects for its application to solar energy conversion," Energy Environ. Sci. 8, 103-125 (2015).

[4] Simon, Y. C. and Weder, C., "Low-power photon upconversion through triplet-triplet annihilation in polymers," J. Mater. Chem. 22, 20817 (2012).

[5] Tayebjee, M. J. Y., McCamey, D. R., and Schmidt, T. W., "Beyond Shockley-Queisser: Molecular approaches to high-efficiency photovoltaics," J. Phys. Chem. Lett. 6, 2367-2378 (2015).

[6] Cheng, Y. Y., Fückel, B., MacQueen, R. W., Khoury, T., Clady, R. G. C. R., Schulze, T. F., Ekins-Daukes, N. J., Crossley, M. J., Stannowski, B., Lips, K., and Schmidt, T. W., "Improving the light-harvesting of amorphous silicon solar cells with photochemical upconversion," Energy Environ. Sci. 5, 6953-6959 (2012). 
[7] Schulze, T. F., Cheng, Y. Y., Fückel, B., MacQueen, R. W., Danos, A., Davis, N. J. L. K., Tayebjee, M. J. Y., Khoury, T., Clady, R. G. C. R., Ekins-Daukes, N. J., Crossley, M. J., Stannowski, B., Lips, K., and Schmidt, T. W., "Photochemical upconversion enhanced solar cells: Effect of a back reflector," Aust. J. Chem. 65, 480-485 (2012).

[8] Schulze, T. F., Cheng, Y. Y., Khoury, T., Crossley, M. J., Stannowski, B., Lips, K., and Schmidt, T. W., "Micro-optical design of photochemical upconverters for thin-film solar cells," J. Photon. Energ. 3(1), 034598-034598 (2013).

[9] Schulze, T. F., Czolk, J., Cheng, Y.-Y., Fückel, B., MacQueen, R. W., Khoury, T., Crossley, M. J., Stannowski, B., Lips, K., Lemmer, U., et al., "Efficiency enhancement of organic and thin-film silicon solar cells with photochemical upconversion," J. Phys. Chem. C 116(43), 22794-22801 (2012).

[10] Nattestad, A., Cheng, Y. Y., MacQueen, R. W., Schulze, T. F., Thompson, F. W., Mozer, A. J., Fückel, B., Khoury, T., Crossley, M. J., Lips, K., Wallace, G. G., and Schmidt, T. W., "Dye-sensitized solar cell with integrated triplet-triplet annihilation upconversion system," J. Phys. Chem. Lett. 4, 2073-2078 (2013).

[11] Khnayzer, R. S., Blumhoff, J., Harrington, J. A., Haefele, A., Deng, F., and Castellano, F. N., "Upconversion-powered photoelectrochemistry," Chem. Commun. 48, 209-211 (2012).

[12] Wang, B., Sun, B., Wang, X., Ye, C., Ding, P., Liang, Z., Chen, Z., Tao, X., and Wu, L., "Efficient triplet sensitizers of palladium(II) tetraphenylporphyrins for upconversion-powered photoelectrochemistry," J. Phys. Chem. C 118(3), 1417-1425 (2014).

[13] Ye, C., Wang, B., Hao, R., Wang, X., Ding, P., Tao, X., Chen, Z., Liang, Z., and Zhou, Y., "Oil-inwater microemulsion: an effective medium for triplet-triplet annihilated upconversion with efficient triplet acceptors," J. Mater. Chem. C 2, 8507-8514 (2014).

[14] Liu, Q., Yang, T., Feng, W., and Li, F., "Blue-emissive upconversion nanoparticles for low-power-excited bioimaging in vivo," J. Am. Chem. Soc. 134(11), 5390-5397 (2012).

[15] Liu, Q., Yin, B., Yang, T., Yang, Y., Shen, Z., Yao, P., and Li, F., "A general strategy for biocompatible, high-effective upconversion nanocapsules based on triplettriplet annihilation," J. Am. Chem. Soc. 135, 5029-5037 (2013).

[16] Liu, Q., Feng, W., Yang, T., Yi, T., and Li, F., "Upconversion luminescence imaging of cells and small animals," Nature Protocols 8, 2033-2044 (2013).

[17] Askes, S. H. C., Mora, N. L., Harkes, R., Koning, R. I., Koster, B., Schmidt, T., Kros, A., and Bonnet, S., "Imaging the lipid bilayer of giant unilamellar vesicles using red-to-blue light upconversion," Chem. Commun. 51, 9137-9140 (2015).

[18] Gai, S., Yang, P., Li, C., Wang, W., Dai, Y., Niu, N., and Lin, J., "Synthesis of magnetic, up-conversion luminescent, and mesoporous coreshell-structured nanocomposites as drug carriers," Adv. Funct. Mater. 20(7), 1166-1172 (2010).

[19] Simon, Y. C., Bai, S., Sing, M. K., Dietsch, H., Achermann, M., and Weder, C., "Low-power upconversion in dye-doped polymer nanoparticles," Macromol. Rapid Comm. 33, 498-502 (2012).

[20] Kim, J.-H. and Kim, J.-H., "Encapsulated triplet-triplet annihilation-based upconversion in the aqueous phase for sub-band-gap semiconductor photocatalysis," J. Am. Chem. Soc. 134, 17478-17481 (2012).

[21] Schmidt, T. W. and Castellano, F. N., "Photochemical upconversion: The primacy of kinetics," J. Phys. Chem. Lett. 5(22), 4062-4072 (2014).

[22] Cheng, Y., Fückel, B., Khoury, T., Clady, R. G. C. R., Tayebjee, M. J. Y., Ekins-Daukes, N. J., Crossley, M. J., and Schmidt, T. W., "Kinetic analysis of photochemical upconversion by triplet-triplet annihilation: Beyond any spin statistical limit," J. Phys. Chem. Lett. 1, 1795-1799 (2010).

[23] Cheng, Y., Khoury, T., Clady, R. G. C. R., Tayebjee, M. J. Y., Ekins-Daukes, N. J., Crossley, M. J., and Schmidt, T. W., "On the efficiency limit of triplet-triplet annihilation for photochemical upconversion," Phys. Chem. Chem. Phys. 12, 66-71 (2010).

[24] Hoseinkhani, S., Tubino, R., Meinardi, F., and Monguzzi, A., "Achieving the photon up-conversion thermodynamic yield upper limit by sensitized triplet-triplet annihilation," Phys. Chem. Chem. Phys. 17, 40204024 (2015). 
[25] MacQueen, R. W., Cheng, Y. Y., Danos, A. N., Lips, K., and Schmidt, T. W., "Action spectrum experiment for the measurement of incoherent photon upconversion efficiency under sun-like excitation," $R S C A d v .4$, 52749-52756 (2014).

[26] Monguzzi, A., Mezyk, J., Scotognella, F., Tubino, R., and Meinardi, F., "Upconversion-induced fluorescence in multicomponent systems: Steady-state excitation power threshold," Phys. Rev. B 78, 5 (2008).

[27] Blacket, S., A Molecular Understanding of Photochemical Upconverters for Highly Efficient Solar Cells, hons., The University of Adelaide (2013).

[28] Singh-Rachford, T. N. and Castellano, F. N., "Supra-nanosecond dynamics of a red-to-blue photon upconversion system," Inorg. Chem. 48, 2541-2548 (2009).

[29] Danos, A., MacQueen, R. W., Cheng, Y. Y., Dvok, M., Darwish, T. A., McCamey, D. R., and Schmidt, T. W., "Deuteration of perylene enhances photochemical upconversion efficiency," J. Phys. Chem. Lett. 6(15), 3061-3066 (2015).

[30] Hirata, S., Totani, K., Zhang, J., Yamashita, T., Kaji, H., Marder, S. R., Watanabe, T., and Adachi, C., "Efficient persistent room temperature phosphorescence in organic amorphous materials under ambient conditions," Adv. Funct. Mater. 23(27), 3386-3397 (2013).

[31] Jankus, V., Snedden, E. W., Bright, D. W., Whittle, V. L., Williams, J. A. G., and Monkman, A., "Energy upconversion via triplet fusion in super yellow PPV films doped with palladium tetraphenyltetrabenzoporphyrin: a comprehensive investigation of exciton dynamics," Adv. Funct. Mater. 23(3), 384-393 (2013).

[32] Shimizu, Y. and Azumi, T., "Mechanism of external heavy atom effect on intersystem crossing in fluid solutions. analysis based on fluorescence decay data," J. Phys. Chem. 86(1), 22-26 (1982).

[33] MacQueen, R. W., Schulze, T. F., Khoury, T., Cheng, Y. Y., Stannowski, B., Lips, K., Crossley, M. J., and Schmidt, T., "Nanostructured upconverters for improved solar cell performance," Proc. SPIE 8824, 882408 (2013). 